\title{
A GENERALIZATION OF THE ALEKSANDROV OPERATOR AND ADJOINTS OF WEIGHTED COMPOSITION OPERATORS
}

\author{
EVA A. GALLARDO-GUTIÉRREZ AND JONATHAN R. PARTINGTON
}

\begin{abstract}
A generalization of the Aleksandrov operator is provided, in order to represent the adjoint of a weighted composition operator on $\mathcal{H}^{2}$ by means of an integral with respect to a measure. In particular, we show the existence of a family of measures which represents the adjoint of a weighted composition operator under fairly mild assumptions, and we discuss not only uniqueness but also the generalization of Aleksandrov-Clark measures which corresponds to the unweighted case, that is, to the adjoint of composition operators.
\end{abstract}

\section{INTRODUCTION}

Let $\mathbb{D}$ denote the open unit disk of the complex plane, $\mathbb{T}$ its boundary and $m$ the normalized arc-length measure on $\mathbb{T}$. For $1 \leq p<\infty$, let $\mathcal{H}^{p}$ be the classical Hardy space, that is, the space of holomorphic functions $f$ on $\mathbb{D}$ for which the norm

$$
\|f\|_{p}=\left(\sup _{0 \leq r<1} \int_{\mathbb{T}}|f(r \zeta)|^{p} d m(\zeta)\right)^{1 / p}
$$

is finite. The space consisting of bounded analytic functions on $\mathbb{D}$ will be denoted by $\mathcal{H}^{\infty}$.

Given two analytic functions $h$ and $\varphi$ on $\mathbb{D}$ such that $\varphi(\mathbb{D}) \subset \mathbb{D}$, it is possible to define a linear map $W_{h, \varphi}$ by

$$
W_{h, \varphi} f(z)=h(z) f(\varphi(z)) \quad \text { for } \quad f \in \mathcal{H}^{p} .
$$

If $W_{h, \varphi} f$ also lies in $\mathcal{H}^{p}$, then we say that $f$ belongs to the domain of $W_{h, \varphi}$, which will denoted by $\mathcal{D}\left(W_{h, \varphi}\right)$. The linear map in (1) is called a weighted composition operator. In some instances, we will write $C_{\varphi}=W_{1, \varphi}$ to denote the standard unweighted composition operator.

As a consequence of the Littlewood Subordination Theorem [9], it is straightforward that the condition $h \in \mathcal{H}^{\infty}$ is always sufficient for boundedness of $W_{h, \varphi}$. By considering the image of the constant functions, it is clear that $h \in \mathcal{H}^{p}$ is a necessary condition.

Date: April 2010; final revision February 2012.

1991 Mathematics Subject Classification. Primary: 47B33, 30D55.

Key words and phrases. Aleksandrov operator, Aleksandrov-Clark measures, Weighted composition operators.

The authors are partially supported by Plan Nacional I+D grant no. MTM2010-16679 and Gobierno de Aragón research group Análisis Matemático y Aplicaciones, ref. DGA E-64. 
In 1990, D. Sarason [16] described composition operators $W_{1, \varphi}$ as integral operators acting on the unit circle. Indeed, if $\mathcal{M}$ denotes the space of all finite complex Borel measures on $\mathbb{T}$ endowed with the total variation norm, Sarason's approach was as follows: if $\mu \in \mathcal{M}$ is given, then the Poisson integral

$$
P[\mu](z)=\int_{\mathbb{T}} P_{z}(\zeta) d \mu(\zeta)
$$

where $P_{z}(\zeta)=\frac{1-|z|^{2}}{|\zeta-z|^{2}}, \quad(\zeta \in \mathbb{T})$, is the Poisson kernel for $z \in \mathbb{D}$, defines a harmonic function on $\mathbb{D}$. Consequently the function $P[\mu] \circ \varphi$ is also harmonic, and therefore it is the Poisson integral of a unique measure $\nu \in \mathcal{M}$. Thus it makes sense to define

$$
W_{1, \varphi} \mu=\nu
$$

It holds that $W_{1, \varphi}: \mathcal{M} \rightarrow \mathcal{M}$ is bounded and, furthermore, that $W_{1, \varphi}$ restricts to a bounded operator $L^{p}(\mathbb{T}) \rightarrow L^{p}(\mathbb{T})$, where $L^{p}(\mathbb{T})=L^{p}(\mathbb{T}, m)$ for $1 \leq p \leq \infty$. Moreover, viewing the Hardy space $\mathcal{H}^{p}$ as a subspace of $L^{p}(\mathbb{T})$ (through the non-tangential boundary values of $\mathcal{H}^{p}$ functions), the restriction of $W_{1, \varphi}$ to $\mathcal{H}^{p}$ coincides with the standard definition of $W_{1, \varphi}$.

Later, Cima and Matheson (see [2]) proved that if $\varphi(0)=0$, then $W_{1, \varphi}: \mathcal{M} \rightarrow \mathcal{M}$ is the adjoint of the Aleksandrov operator $A_{\varphi}$, considered first by A. B. Aleksandrov in [1] and defined on the space of continuous functions on the circle $\mathcal{C}(\mathbb{T})$ by

$$
A_{\varphi} f(\alpha)=\int_{\mathbb{T}} f(\zeta) d \tau_{\varphi, \alpha}(\zeta), \quad(\alpha \in \mathbb{T}),
$$

where $\tau_{\varphi, \alpha}$ is the positive measure with Poisson integral

$$
P\left[\tau_{\varphi, \alpha}\right](z)=\Re\left(\frac{\alpha+\varphi(z)}{\alpha-\varphi(z)}\right), \quad(z \in \mathbb{D}) .
$$

Recall that the family of measures $\left\{\tau_{\varphi, \alpha}: \alpha \in \mathbb{T}\right\}$ in (3) are called the AleksandrovClark measures associated to $\varphi$. Recently, Aleksandrov-Clark measures have played an important role in connection with composition operators (see [12], [13], or [7] for instance). Nevertheless, these measures have important applications in other areas of analysis (we refer the reader to the lecture notes [15], the book [3] and the surveys [10, 14], for more on the subject).

Let us remark here that the Aleksandrov operator takes $L^{p}(\mathbb{T})$ boundedly into itself for all $1 \leq p \leq \infty$. The key point is the so-called Disintegration Theorem which states that for all $f \in L^{1}(\mathbb{T})$, one has $f \in L^{1}\left(\mathbb{T}, \tau_{\varphi, \alpha}\right)$ and the equality

$$
m=\int_{\mathbb{T}} \tau_{\varphi, \alpha} d m(\alpha)
$$

holds true in the following sense

$$
\int_{\mathbb{T}} f d m=\int_{\mathbb{T}}\left(\int_{\mathbb{T}} f d \tau_{\varphi, \alpha}\right) d m(\alpha)
$$


for all $f \in L^{1}(\mathbb{T})$. Hence, for any $f \in L^{1}(\mathbb{T})$ the expression defined in (2) is welldefined for $m$-almost every $\alpha \in \mathbb{T}$. Using the Disintegration Theorem and the fact that $\left\{\tau_{\varphi, \alpha}: \alpha \in \mathbb{T}\right\}$ are positive measures, it is easy to check the boundedness of $A_{\varphi}$ in $L^{1}(\mathbb{T})$. The boundedness of $A_{\varphi}$ in $L^{\infty}(\mathbb{T})$ is trivial and the rest follows e.g, by interpolation (see $[15$, Theorem 4.1] for more details).

Furthermore, if $\varphi(0)=0$ then $W_{1, \varphi}: L^{p}(\mathbb{T}) \rightarrow L^{p}(\mathbb{T}),(1 \leq p<\infty)$ is the adjoint of the Aleksandrov operator acting on $L^{q}(\mathbb{T})$, where $\frac{1}{p}+\frac{1}{q}=1$ (see [15, Section 5]).

The aim of this paper is to extend, in some sense, the Aleksandrov operator in order to represent the adjoint of a weighted composition operator in $\mathcal{H}^{2}$ by means of an integral with respect to a measure.

Note that in the easiest case when $h \in \mathcal{H}^{\infty}$ and $\varphi(0)=0$, in which $W_{h, \varphi}$ is just the product of the analytic Toeplitz operator $T_{h}$ and the composition operators $C_{\varphi}$, one deduces taking into account what we said before that the adjoint of $W_{h, \varphi}$ in $L^{2}(\mathbb{T})$ should be given by the integral operator:

$$
f \in L^{2}(\mathbb{T}) \rightarrow \int_{\mathbb{T}} f(\zeta) \overline{h(\zeta)} d \tau_{\varphi, \alpha}(\zeta)
$$

Nevertheless, it is not so clear that when $W_{h, \varphi}$ acts on $\mathcal{H}^{2}$ (identified with a closed subspace of $L^{2}(\mathbb{T})$ through the non-tangential boundary values of the $\mathcal{H}^{2}$ functions), that the integral operator (the candidate for the adjoint of $W_{h, \varphi}$ ):

$$
f \in \mathcal{H}^{2} \rightarrow \int_{\mathbb{T}} \mathbb{P}_{L^{2}(\mathbb{T}) \rightarrow \mathcal{H}^{2}}(f \bar{h})(\zeta) d \tau_{\varphi, \alpha}(\zeta),
$$

may be expressed by means of an integral with respect to a measure; here $\mathbb{P}=\mathbb{P}_{L^{2}(\mathbb{T}) \rightarrow \mathcal{H}^{2}}$ denotes the Riesz projection from $L^{2}(\mathbb{T})$ to $\mathcal{H}^{2}$, which is continuous. Indeed, one of the main difficulties we will find and which will make the problem harder than in the unweighted case $W_{1, \varphi}$ is, simply the well-known fact that the harmonic extension of a product of functions is not in general the product of the harmonic extensions, unless they are analytic.

Another difficulty that we shall address is that, even in the case when $\varphi$ is the identity, we may only conclude that $W_{h, \varphi}^{*}$ (which is the Toeplitz operator $T_{\bar{h}}$ ) only maps the disc algebra into itself for a restricted class of symbols $h$.

In Section 2, we will prove that under these circumstances the adjoint of a weighted composition operator $W_{h, \varphi}$ in $\mathcal{H}^{2}$ may be represented by means of a family of finite complex measures $\left\{\tau_{h, \varphi, \alpha}: \alpha \in \mathbb{T}\right\}$. We will show that, in principle, $\tau_{h, \varphi, \alpha}$ is not uniquely determined (it will be only determined up to an absolutely continuous part $\bar{g} d m$, where $\left.g \in \mathcal{H}_{0}^{1}=\left\{f \in \mathcal{H}^{1}: f(0)=0\right\}\right)$. Nevertheless, imposing a condition of minimality in norm in order to obtain uniqueness, we will prove that the family $\left\{\tau_{h, \varphi, \alpha}: \alpha \in \mathbb{T}\right\}$ is the right generalization of the Aleksandrov-Clark measures in the sense that they coincide whenever $h \equiv 1$. We will close the section with a Disintegration Theorem for the measures $\left\{\tau_{h, \varphi, \alpha}: \alpha \in \mathbb{T}\right\}$ similar to that stated before for the Aleksandrov-Clark measures. 
If given any Borel measure $\mu$ on $\mathbb{T}$, we write $\mu=\mu^{a} d m+\mu^{s}$ for the Lebesgue decomposition of $\mu$, where $\mu^{a}$ is the density of the absolutely continuous part, and $\mu^{s}$ is singular, in Section 3 we will identify the atoms of $\tau_{h, \varphi, \alpha}^{s}$. As in the case of the Aleksandrov-Clark measures, $\tau_{h, \varphi, \alpha}^{s}$ will be closely related to those points on $\mathbb{T}$ where the angular derivative of $\varphi$ exists (finitely).

Finally, we will discuss compactness of weighted composition operators in connection with the family of measures $\left\{\tau_{h, \varphi, \alpha}: \alpha \in \mathbb{T}\right\}$, showing that if $W_{h, \varphi}$ is compact in $\mathcal{H}^{2}$ (or even in $\mathcal{H}^{1}$ ), then $\tau_{h, \varphi, \alpha}^{s}=0$ for any $\alpha \in \mathbb{T}$, generalizing a previous result proved by Sarason [16] in the setting of composition operators.

\section{A family of measures Associated to the Adjoint of $W_{h, \varphi}$}

In this section, we will show the existence of a family of measures which will represent the adjoint of a weighted composition operator, discussing uniqueness as well as the fact that they generalize Aleksandrov-Clark measures in the unweighted case.

Let us begin by recalling that the reproducing kernels $k_{w}$ for $\mathcal{H}^{2}$ are defined for $w \in \mathbb{D}$ by

$$
k_{w}(z)=\frac{1}{1-\bar{w} z}, \quad(z \in \mathbb{D})
$$

and satisfy $\left\langle f, k_{w}\right\rangle=f(w)$ for $f \in \mathcal{H}^{2}$. It is quite straightforward to show that the adjoint of a bounded $W_{h, \varphi}$ on $\mathcal{H}^{2}$ satisfies

$$
W_{h, \varphi}^{*} k_{w}=\overline{h(w)} k_{\varphi(w)}, \quad(w \in \mathbb{D}) .
$$

The next lemma will be useful in our approach to prove the existence of a family of measures representing $W_{h, \varphi}^{*}$. Before stating it, recall that the Riesz projection $\mathbb{P}_{L^{2}(\mathbb{T}) \rightarrow \mathcal{H}^{2}}$ defined on $L^{2}(\mathbb{T})$ by

$$
\mathbb{P}_{L^{2}(\mathbb{T}) \rightarrow \mathcal{H}^{2}} f(z)=\int_{\mathbb{T}} \frac{f(\zeta)}{1-\bar{\zeta} z} d m(\zeta), \quad(z \in \mathbb{D})
$$

is continuous from $L^{2}(\mathbb{T})$ to $\mathcal{H}^{2}$. Moreover, $L^{2}(\mathbb{T})$ may be decomposed in the following way:

$$
L^{2}(\mathbb{T})=\mathcal{H}^{2} \oplus \overline{\mathcal{H}_{0}^{2}}
$$

where $\mathcal{H}_{0}^{2}=\left\{f \in \mathcal{H}^{2}: f(0)=0\right\}$. Note that in the above identity we are identifying $\mathcal{H}^{2}$ through the non-tangential boundary values of the $\mathcal{H}^{2}$ functions.

Lemma 2.1. Suppose that $W_{h, \varphi}$ defines a bounded operator on $\mathcal{H}^{2}$. Then $W_{h, \varphi}$ defines a bounded operator on each of the spaces $L^{2}(\mathbb{T})$ and $\mathcal{H}^{1}$. Moreover, if $W_{h, \varphi}$ is a compact operator on $\mathcal{H}^{2}$, then it is compact when regarded as an operator on each of $L^{2}(\mathbb{T})$ and $\mathcal{H}^{1}$.

Proof. First, let us assume that $W_{h, \varphi}$ is a bounded operator on $\mathcal{H}^{2}$. Now, for $f \in L^{2}(\mathbb{T})$, we may write $f=f_{+}+f_{-}$, where $f_{+} \in \mathcal{H}^{2}$ and $f_{-} \in \overline{\mathcal{H}_{0}^{2}}$. Now

$$
\left\|W_{h, \varphi} f\right\|_{2} \leq\left\|W_{h, \varphi} f_{+}\right\|_{2}+\left\|W_{h, \varphi} f_{-}\right\|_{2},
$$


and

$$
\left\|W_{h, \varphi} f_{-}\right\|_{2}=\left\|h\left(f_{-} \circ \varphi\right)\right\|_{2}=\left\|h\left(\overline{f_{-}} \circ \varphi\right)\right\|_{2} \leq\left\|W_{h, \varphi}\right\|\left\|f_{-}\right\|_{2},
$$

from which we conclude easily that $W_{h, \varphi}$ is bounded on $L^{2}(\mathbb{T})$.

Next, if $f \in \mathcal{H}^{1}$ we may use the Riesz factorization theorem [8, p. 84] to write $f=g h$, where $g, h \in \mathcal{H}^{2}$ and $\|f\|_{1}=\|g\|_{2}\|h\|_{2}$. Then

$$
W_{h, \varphi} f=\left(W_{h, \varphi} g\right)\left(C_{\varphi} h\right),
$$

and so

$$
\left\|W_{h, \varphi} f\right\|_{1} \leq\left\|W_{h, \varphi}\right\|_{2}\left\|C_{\varphi}\right\|_{2}\|g\|_{2}\|h\|_{2}=\left\|W_{h, \varphi}\right\|_{2}\left\|C_{\varphi}\right\|_{2}\|f\|_{1},
$$

from which we obtain boundedness on $\mathcal{H}^{1}$.

The compactness results are proved similarly. If $\left(f_{n}\right)$ is a bounded a sequence in $L^{2}(\mathbb{T})$, we may write each $f_{n}=f_{n+}+f_{n-}$, where $f_{n+} \in \mathcal{H}^{2}$ and $f_{n-} \in \overline{\mathcal{H}_{0}^{2}}$. We may then pass to a subsequence and relabel it to assume that both $\left(h f_{+n} \circ \varphi\right)$ and $\left(h \overline{f_{-n}} \circ \varphi\right)$ converge, which establishes the convergence of $\left(h f_{n} \circ \varphi\right)$, by a calculation similar to the one above showing boundedness.

Likewise, for a bounded sequence $\left(f_{n}\right)$ in $\mathcal{H}^{1}$, we write each $f_{n}=g_{n} h_{n}$ with $\left\|f_{n}\right\|_{1}=\left\|g_{n}\right\|_{2}\left\|h_{n}\right\|_{2}$, and use the identity $W_{h, \varphi} f_{n}=\left(W_{h, \varphi} g_{n}\right)\left(C_{\varphi} h_{n}\right)$ to obtain the desired conclusion. This proves the lemma.

In the case of unweighted composition operators, it was shown by Shapiro and Sundberg [17] that compactness on $\mathcal{H}^{2}$ and compactness on $L^{1}(\mathbb{T})$ are equivalent. Whether it is true for weighted composition operators that boundedness or compactness on $\mathcal{H}^{2}$ implies the same for $L^{1}(\mathbb{T})$ is unknown: the converse is easily seen to be false, as suitable examples can be constructed by taking a weight that lies in $\mathcal{H}^{1}$ but not $\mathcal{H}^{2}$. However, it is possible to derive necessary and sufficient conditions for boundedness and compactness on $L^{1}(\mathbb{T})$ by regarding $W_{h, \varphi}$ as an integral operator, an idea introduced by Sarason [16] in the unweighted case.

Proposition 2.2. The operator $W_{h, \varphi}$ is bounded on $L^{1}(\mathbb{T})$ if and only if

$$
A:=\sup _{0 \leq r<1, \zeta \in \mathbb{T}} \int_{\mathbb{T}}\left|K_{r}(\xi, \zeta)\right| d m(\xi)<\infty,
$$

where for suitable $\xi, \zeta \in \mathbb{C}$ we define

$$
K_{r}(\xi, \zeta)=h(\xi) \frac{1-|r \varphi(\xi)|^{2}}{|\zeta-r \varphi(\xi)|^{2}} .
$$

Moreover, if the operator is bounded, then its norm is $A$.

Further if $W_{h, \varphi}$ is bounded, then it is compact on $L^{1}(\mathbb{T})$ if and only if $|\varphi(\xi)|<1$ a.e. on $\mathbb{T}$ and given any $\varepsilon>0$ there exists $\delta>0$ such that

$$
\sup _{\eta, \zeta \in \mathbb{T},|\eta-1|<\delta} \int_{\mathbb{T}}\left|K_{1}(\xi, \zeta)-K_{1}(\eta \xi, \zeta)\right| d m(\xi)<\varepsilon .
$$


Proof. Using the ideas of [16], we note that if $|\varphi(\xi)|<1$ a.e., then the weighted composition operator may be expressed, using the Poisson extension of $f \circ \varphi$ for $f \in L^{1}(\mathbb{T})$ as

$$
W_{h, \varphi} f(\xi)=h(\xi) C_{\varphi} f(\xi)=\int_{\mathbb{T}} K_{1}(\xi, \zeta) f(\zeta) d m(\zeta),
$$

from which we have

$$
\left\|W_{h, \varphi}\right\|=\sup _{\zeta \in \mathbb{T}} \int_{\mathbb{T}}\left|K_{1}(\xi, \zeta)\right| d m(\xi)
$$

using the standard formula for the norm of an integral operator on $L^{1}(\mathbb{T})$. The general case is obtained on considering the operators $W_{h, r \varphi}$, which tend strongly to $W_{h, \varphi}$ as $r \rightarrow 1$.

For compactness, it is necessary that $|\varphi(\xi)|<1$ a.e., as otherwise, on writing $e_{n}(z)=z^{n}$ for $n=1,2,3, \ldots$, we see that the sequence of images $\left(W_{h, \varphi} e_{n}\right)$ cannot tend to zero in norm. In this case we may use a standard compactness criterion for integral operators on $L^{1}(\mathbb{T})$, which may be found, for example, in [6, Cor. 5.1].

Recall that the Cauchy transform, $\mathcal{K} \mu$, of a finite complex Borel measure $\mu \in \mathcal{M}$ is defined for $z \in \mathbb{D}$ by

$$
(\mathcal{K} \mu)(z)=\int_{\mathbb{T}} \frac{d \mu(\zeta)}{1-\bar{\zeta} z} .
$$

The space of all Cauchy transforms $\mathcal{K} \mu$ will be denoted by $\mathfrak{K}$. An analytic function $\Phi$ in $\mathbb{D}$ is called a multiplier of the space of the Cauchy transforms if

$$
f \in \mathfrak{K} \Rightarrow \Phi f \in \mathfrak{K} .
$$

The set of multipliers of $\mathfrak{K}$ will be denoted by $\mathcal{M}(\mathfrak{K})$. If $\Phi \in \mathcal{M}(\mathfrak{K})$, the multiplication operator

$$
M_{\Phi}: \mathfrak{K} \rightarrow \mathfrak{K}, \quad M_{\Phi} f=\Phi f
$$

is well-defined and bounded on $\mathfrak{K}$ when this space is given the quotient norm

$$
\|f\|_{\mathfrak{K}}=\inf \{\|\mu\|: \mu \in \mathcal{M}, \mathcal{K} \mu=f\},
$$

inherited from $\mathcal{M}$. We refer the reader to [3, Chapter 6] for more properties and results on multipliers of $\mathfrak{K}$. In particular, it is known from [3, Prop. 6.1.5] that the following conditions are equivalent for $h \in \mathcal{H}^{\infty}$ :

(a) $h \in M(\mathfrak{K})$;

(b) the Toeplitz operator $f \mapsto T_{\bar{h}} f=\mathbb{P}_{L^{2}(\mathbb{T}) \rightarrow \mathcal{H}^{2}} \bar{h} f$ maps $\mathcal{H}^{\infty}$ boundedly into itself;

(c) $T_{\bar{h}}$ maps the disc algebra $\mathcal{A}(\mathbb{D})$ boundedly into itself.

It is also known from [3, Thm. 6.4.1] that for $h \in \mathcal{H}^{\infty}$ given by $h(z)=\sum_{n=0}^{\infty} h_{n} z^{n}$ the condition

$$
\sum_{n=2}^{\infty}\left|h_{n}\right| \log n<\infty
$$

is a sufficient condition for $h$ to lie in $\mathcal{M}(\mathfrak{K})$. 
The significance of the above remarks in the context of weighted composition operators is explained by the following observation.

Lemma 2.3. Suppose that the Toeplitz operator $T_{\bar{h}}$ maps $\mathcal{A}(\mathbb{D})$ boundedly into itself. Then for any analytic self-map $\varphi: \mathbb{D} \rightarrow \mathbb{D}$ the adjoint of the weighted composition operator $W_{h, \varphi}$ on $\mathcal{H}^{2}$ maps $\mathcal{A}(\mathbb{D})$ boundedly into itself.

Proof. For $f \in \mathcal{A}(\mathbb{D})$, we have $W_{h, \varphi}^{*} f=C_{\varphi}^{*} T_{\bar{h}} f$. Consider the operator $C_{\varphi}^{*}$ acting on $\mathcal{A}(\mathbb{D})$. Observe that a finite linear combination $\sum_{j=1}^{N} a_{j} k_{w_{j}}$ of reproducing kernels is mapped into $\sum_{j=1}^{N} a_{j} k_{\varphi\left(w_{j}\right)}$, which also lies in $\mathcal{A}(\mathbb{D})$. Since $C_{\varphi}^{*}$ is bounded in the uniform norm, and since the finite linear combinations of reproducing kernels are dense in $\mathcal{A}(\mathbb{D})$, one deduces that $C_{\varphi}^{*}$ preserves $\mathcal{A}(\mathbb{D})$.

Alternatively, if $\varphi(0)=0, C_{\varphi}^{*}$ is just the Aleksandrov operator $A_{\varphi}$, which is well-known to preserve analyticity and to be a contraction in the uniform norm [15, Thm. 4.1]; in the general case one can compose with a linear fractional transformation, for which the adjoint of the associated composition operator is easily seen to preserve $\mathcal{A}(\mathbb{D})$ (cf. [4]).

Suppose that $W_{h, \varphi}$ defines a bounded operator on $\mathcal{H}^{2}$. Having in mind Lemma 2.1, and in order to get a family of measures associated to $W_{h, \varphi}^{*}$, one would be tempted to check if $W_{h, \varphi}^{*}$ takes the the space $\mathcal{C}(\mathbb{T})$ of continuous functions on $\mathbb{T}$ into itself (here we are again identifying functions through its non-tangential boundary values). However, since $W_{h, \varphi}^{*}$ includes an implicit Riesz projection, this is not a fruitful line of enquiry.

Suppose that $W_{h, \varphi}^{*}$ is bounded on $\mathcal{A}(\mathbb{D})$. If $\alpha \in \mathbb{T}$, then the mapping

$$
f \in \mathcal{A}(\mathbb{D}) \mapsto W_{h, \varphi}^{*} f(\alpha)
$$

is bounded on $\mathcal{A}(\mathbb{D})$, and thus there exists a measure $\mu_{h, \varphi, \alpha} \in \mathcal{M}(\mathbb{T})$, not unique, such that

$$
\overline{h(z)} k_{\varphi(z)}(\alpha)=W_{h, \varphi}^{*} k_{z}(\alpha)=\int_{0}^{2 \pi} k_{z}\left(e^{i \theta}\right) d \mu_{h, \varphi, \alpha}(\theta)=\int_{0}^{2 \pi} \frac{d \mu_{h, \varphi, \alpha}(\theta)}{1-\bar{z} e^{i \theta}},
$$

for every $z \in \mathbb{D}$. In order to express this in the language of Cauchy transforms, we take the complex conjugate of the measure, and write

$$
h(z) \overline{k_{\varphi(z)}(\alpha)}=\frac{h(z)}{1-\bar{\alpha} \varphi(z)}=\int_{\mathbb{T}} \frac{d \nu_{h, \varphi, \alpha}(\zeta)}{1-\bar{\zeta} z}=\left(\mathcal{K} \nu_{h, \varphi, \alpha}\right)(z) .
$$

That is, it is the Cauchy transform of the measure $\nu_{h, \varphi, \alpha}$. According to [3, Prop. 4.1.4], although $\nu_{h, \varphi, \alpha}$ is only determined up to an absolutely continuous part $\bar{g} d m$, where $g \in \mathcal{H}_{0}^{1}$ and $m$ is Lebesgue measure, there is a unique measure of minimal norm such that (4) holds.

Definition 2.1. Suppose that $W_{h, \varphi}^{*}$ is bounded on $\mathcal{A}(\mathbb{D})$. For $\alpha \in \mathbb{T}$ the Borel measure $\tau_{h, \varphi, \alpha}$ on $\mathbb{T}$ is defined to be the minimal-norm measure such that

$$
\int_{\mathbb{T}} \frac{1}{1-\bar{\zeta} z} d \tau_{h, \varphi, \alpha}(\zeta)=\frac{h(z)}{1-\bar{\alpha} \varphi(z)}
$$

for all $z \in \mathbb{D}$. 
Note that we have the identity

$$
\int_{\mathbb{T}} \overline{f(\zeta)} d \tau_{h, \varphi, \alpha}(\zeta)=\overline{W_{h, \varphi}^{*} f(\alpha)}
$$

for all $f \in \mathcal{A}(\mathbb{D})$, since it holds for $f=k_{z}$.

Remark 2.2. A detailed and explicit account of the extremal problem inf ${ }_{h \in \overline{\mathcal{H}_{0}^{1}}}\|g-h\|_{1}$, in the case when $g$ is a polynomial, can be found in [8, p. 169, Ex. 3].

Observe that we have a Herglotz-like formula as in the case of Alexandrov-Clark measures based on the identity:

$$
\int_{0}^{2 \pi} \frac{e^{i \theta}+z}{e^{i \theta}-z} d \tau_{h, \varphi, \alpha}(\theta)=\int_{0}^{2 \pi}\left[-1+\frac{2}{1-e^{-i \theta} z}\right] d \tau_{h, \varphi, \alpha}(\theta) .
$$

We state it as a proposition:

Proposition 2.4. Suppose that $W_{h, \varphi}^{*}$ is bounded on $\mathcal{A}(\mathbb{D})$. Let $\tau_{h, \varphi, \alpha}$ be the Borel measure given by Definition 2.1. Then

$$
\int_{0}^{2 \pi} \frac{e^{i \theta}+z}{e^{i \theta}-z} d \tau_{h, \varphi, \alpha}(\theta)=-\frac{h(0)}{1-\bar{\alpha} \varphi(0)}+\frac{2 h(z)}{1-\bar{\alpha} \varphi(z)} .
$$

Example 2.5. Let $\varphi(z)=z^{2}$. In the unweighted case $h(z)=1$ we have

$$
\int_{\mathbb{T}} \frac{d \tau_{h, \varphi, \alpha}(\zeta)}{1-\bar{\zeta} z}=\frac{1}{1-\bar{\alpha} z^{2}}=\frac{1}{2}\left(\frac{1}{1-\bar{\beta} z}+\frac{1}{1+\bar{\beta} z}\right),
$$

where $\beta$ is a square root of $\alpha$. Thus $\tau_{h, \varphi, \alpha}=\frac{1}{2}\left(\delta_{\beta}+\delta_{-\beta}\right)$, where $\delta$ denotes a Dirac point mass, since adding on a continuous part can only increase the norm. This is the standard Aleksandrov-Clark measure.

Example 2.6. Let $\varphi(z)=z^{2}$. A similar computation as before applies for the weight $h(z)=z$, and the corresponding measure, although now complex, is still atomic and concentrated on $\pm \beta$.

The case $h(z)=z^{2}$ is more interesting. We now have

$$
\int_{\mathbb{T}} \frac{d \tau_{h, \varphi, \alpha}(\zeta)}{1-\bar{\zeta} z}=\frac{z^{2}}{1-\bar{\alpha} z^{2}}=-\alpha+\frac{\alpha}{2}\left(\frac{1}{1-\bar{\beta} z}+\frac{1}{1+\bar{\beta} z}\right),
$$

Again the singular part is atomic; the continuous part can be expressed as $-\alpha d \zeta /(2 \pi i \zeta)$, or as $-\alpha d \theta /(2 \pi)$, where $\zeta=e^{i \theta}$, since

$$
\int_{\mathbb{T}} \frac{\bar{\zeta} d \zeta}{1-\bar{\zeta} z}=2 \pi i
$$

This cannot be reduced in norm by adding on an anti-analytic symbol. A similar story holds for $h(z)=z^{3}$, where the continuous part has Radon-Nikodym derivative proportional to $\zeta$. 
With the Example 2.5 at hand, one might ask if the family of measures $\left\{\tau_{h, \varphi, \alpha}: \alpha \in \mathbb{T}\right\}$ given by Definition 2.1 agrees with the Alexandrov-Clark measures when $h \equiv 1$. In other words, if in the unweighted case, the Aleksandrov-Clark measures corresponds to the minimal norm ones. The answer is affirmative and the next lemma is the key of the argument:

Lemma 2.7. Let $f \in L^{1}(\mathbb{T})$ be a function such that $f \geq 0$. Then $\|f+h\|_{1} \geq\|f\|_{1}$ for any $h \in \overline{\mathcal{H}_{0}^{1}}$.

Proof. For any $u \in L^{1}(\mathbb{T})$ and $v \in L^{\infty}(\mathbb{T})$, let us denote by $\langle u, v\rangle_{1, \infty}$ the dual pairing $\int_{\mathbb{T}} u(\zeta) \overline{v(\zeta)} d \zeta$, which gives the dual pair $\left(L^{1}(\mathbb{T}), L^{\infty}(\mathbb{T})\right)$. Then, if $f \in L^{1}(\mathbb{T})$ is a positive function and $h \in \overline{\mathcal{H}_{0}^{1}}$ one has

$$
\langle f+h, 1\rangle_{1, \infty}=\langle f, 1\rangle_{1, \infty}=\|f\|_{1} .
$$

Since $\|f+h\|_{1}=\sup \left\{\left|\langle f+h, v\rangle_{1, \infty}\right|: v \in L^{\infty}(\mathbb{T})\right.$ and $\left.\|v\|_{\infty} \leq 1\right\}$, one easily obtains the statement of the lemma.

With Lemma 2.7 at hand, we have the following

Proposition 2.8. Let $\varphi$ be a holomorphic self-map of $\mathbb{D}$ such that $\varphi(0)=0$. The family of Aleksandrov-Clark measures $\left\{\tau_{\varphi, \alpha}: \alpha \in \mathbb{T}\right\}$ associated to $\varphi$ agrees with the family defined by Definition 2.1 for $h \equiv 1$, i.e. $\left\{\tau_{1, \varphi, \alpha}: \alpha \in \mathbb{T}\right\}$.

Proof. It is clear that $W_{1, \varphi}$ is a bounded operator on $\mathcal{H}^{2}$. Hence, any measure satisfying equation (4) with $h \equiv 1$ can be expressed by:

$$
\nu_{1, \varphi, \alpha}=\tau_{1, \varphi, \alpha}+\bar{g} d m,
$$

for $g \in \mathcal{H}_{0}^{1}$. Now, $\tau_{1, \varphi, \alpha}$ is the Aleksandrov-Clark measure associated to $\varphi$ at $\alpha$ since by definition the Cauchy transform of $\tau_{1, \varphi, \alpha}$ is given by

$$
\left(\mathcal{K} \tau_{1, \varphi, \alpha}\right)(z)=\frac{1}{1-\bar{\alpha} \varphi(z)}, \quad(z \in \mathbb{D}) .
$$

Having in mind that Aleksandrov-Clark measures are positive measures, by Lemma 2.7 one deduces that $\left\|\nu_{1, \varphi, \alpha}\right\| \geq\left\|\tau_{1, \varphi, \alpha}\right\|$.

Remark 2.3. Note that if $\varphi$ is a holomorphic self-map of $\mathbb{D}$ such that $\varphi(0) \neq 0$, then $C_{\varphi}: L^{2}(\mathbb{T}) \rightarrow L^{2}(\mathbb{T})$ does not correspond exactly to the adjoint of the Aleksandrov operator on $L^{2}(\mathbb{T})$ (see $[15$, Section 5]). This explains the hypotheses $\varphi(0)=0$ in Proposition 2.8. Moreover, when $\varphi(0) \neq 0$, the Cauchy transform of the Aleksandrov-Clark measure $\tau_{\varphi, \alpha}$ is given by:

$$
\left(\mathcal{K}_{\tau_{\varphi, \alpha}}\right)(z)=\frac{1}{1-\bar{\alpha} \varphi(z)}+\frac{\left\|\tau_{\varphi, \alpha}\right\|-1}{2}+i \frac{\Im(\overline{\alpha \varphi(0)})}{|\alpha-\varphi(0)|^{2}},
$$

for $z \in \mathbb{D}$ (see Corollary 9.1.7 in [3]). 
Finally, given $W_{h, \varphi}$ a bounded weighted composition operator on $\mathcal{H}^{2}$, we will prove a Disintegration Theorem for the family $\left\{\tau_{h, \varphi, \alpha}: \alpha \in \mathbb{T}\right\}$.

Theorem 2.4 (Disintegration Theorem). Let $W_{h, \varphi}$ be a bounded weighted composition operator on $\mathcal{H}^{2}$ such that $W_{h, \varphi}^{*}$ is bounded on $\mathcal{A}(\mathbb{D})$. Let $\left\{\tau_{h, \varphi, \alpha}: \alpha \in \mathbb{T}\right\}$ be the family of measures associated to $W_{h, \varphi}$, given by Definition 2.1. Then, for any $g \in \mathcal{A}(\mathbb{D})$ it holds that

$$
\int_{\mathbb{T}}\left(\int_{\mathbb{T}} \overline{g(\zeta)} d \tau_{h, \varphi, \alpha}(\zeta)\right) d m(\alpha)=\int_{\mathbb{T}} \overline{g(\zeta)} h(\zeta) d m(\zeta) .
$$

Proof. Let $z \in \mathbb{D}$. Since $\tau_{h, \varphi, \alpha}$ is a complex measure, one has

$$
\int_{\mathbb{T}} \frac{1}{1-\bar{\zeta} z} d \tau_{h, \varphi, \alpha}(\zeta)=\sum_{n=0}^{\infty}\left(\int_{\mathbb{T}} \overline{\zeta^{n}} d \tau_{h, \varphi, \alpha}(\zeta)\right) z^{n} .
$$

On the other hand, $h(z) /(1-\bar{\alpha} \varphi(z))$ for $z \in \mathbb{D}$ is an analytic function on $\mathbb{D}$; so we may write:

$$
\frac{h(z)}{1-\bar{\alpha} \varphi(z)}=h(z)\left[1+\bar{\alpha} \varphi(z)+\bar{\alpha}^{2}(\varphi(z))^{2}+\cdots\right], \quad(z \in \mathbb{D}) .
$$

From (5) and (6) it follows that $\int_{\mathbb{T}} \overline{\zeta^{n}} d \tau_{h, \varphi, \alpha}(\zeta)$ corresponds to the $n$-th coefficient of the Taylor series of

$$
h(z)\left[1+\bar{\alpha} \varphi(z)+\bar{\alpha}^{2}(\varphi(z))^{2}+\cdots\right] .
$$

Integrating with respect to $\alpha \in \mathbb{T}$, we get the $n$-th Taylor coefficient of $h$, that is,

$$
\int_{\mathbb{T}}\left(\int_{\mathbb{T}} \overline{\zeta^{n}} d \tau_{h, \varphi, \alpha}(\zeta)\right) d m(\alpha)=\int_{\mathbb{T}} h(\zeta) \overline{\zeta^{n}} d m(\zeta)
$$

From here, the statement of the theorem follows.

\section{IDENTIFying ATOMS OF The SingulaR PART OF $\tau_{h, \varphi, \alpha}$}

In this section, we examine the points of $\mathbb{T}$ where the measures $\left\{\tau_{h, \varphi, \alpha}: \alpha \in \mathbb{T}\right\}$ associated to a bounded $W_{h, \varphi}$ on $\mathcal{H}^{2}$ have point masses. We will see it is closely related to the those points where $\varphi$ has finite angular derivatives.

Let us recall that if the quotient $(\varphi(z)-\eta) /(z-\zeta)$ has a finite non-tangential limit at $\zeta \in \mathbb{T}$ for some $\eta \in \mathbb{T}$, then this limit is called the angular derivative of $\varphi$ at $\zeta$ and denoted by $\varphi^{\prime}(\zeta)$ :

$$
\varphi^{\prime}(\zeta)=\angle \lim _{z \rightarrow \zeta} \frac{\varphi(z)-\eta}{z-\zeta}
$$

It satisfies $\varphi^{\prime}(\zeta)=\left|\varphi^{\prime}(\zeta)\right| \bar{\zeta} \eta$ with $\eta=\varphi(\zeta)$. Moreover, if $\varphi$ has an angular derivative at $\zeta$, then the Aleksandrov-Clark measure $\tau_{\varphi, \alpha}$ has an atom at $\zeta$, and $\tau_{\varphi, \alpha}(\{\zeta\})=$ $1 /\left|\varphi^{\prime}(\zeta)\right|$. For more properties on angular derivatives of functions and its connection with Aleksandrov-Clark measures, we refer the reader to [3, Chapter 9] and [15]. 
Theorem 3.1. Let $W_{h, \varphi}$ be a bounded weighted composition operator on $\mathcal{H}^{2}$ such that $W_{h, \varphi}^{*}$ is bounded on $\mathcal{A}(\mathbb{D})$. Let $\left\{\tau_{h, \varphi, \alpha}: \alpha \in \mathbb{T}\right\}$ be the family of measures associated to $W_{h, \varphi}$, given by Definition 2.1. Let $\zeta \in \mathbb{T}$. Then $\tau_{h, \varphi, \alpha}(\{\zeta\}) \neq 0$ if and only if

$$
\angle \lim _{z \rightarrow \zeta} \varphi(z)=\alpha \quad \text { and } \quad \angle \lim _{z \rightarrow \zeta}\left(h(z) \frac{\zeta-z}{\alpha-\varphi(z)}\right) \neq 0 .
$$

Furthermore,

$$
\tau_{h, \varphi, \alpha}(\{\zeta\})=\angle \lim _{z \rightarrow \zeta} \frac{\alpha h(z)(\zeta-z)}{\zeta(\alpha-\varphi(z))}
$$

The proof follows the lines of [3, Theorem 9.2.1], which is based on Nevanlinna's proof [11] of the Julia-Carathéodory Theorem.

Proof. By Proposition (2.4), we have that

$$
\int_{0}^{2 \pi} \frac{e^{i \theta}+z}{e^{i \theta}-z} d \tau_{h, \varphi, \alpha}(\theta)=-\frac{h(0)}{1-\bar{\alpha} \varphi(0)}+\frac{2 h(z)}{1-\bar{\alpha} \varphi(z)} .
$$

Let $\zeta \in \mathbb{T}$ fixed. Multiplying both sides of the above formula by $\zeta-z$ we get

$$
\int_{0}^{2 \pi}\left(e^{i \theta}+z\right) \frac{\zeta-z}{e^{i \theta}-z} d \tau_{h, \varphi, \alpha}(\theta)=-(\zeta-z) \frac{h(0)}{1-\bar{\alpha} \varphi(0)}+(\zeta-z) \frac{2 h(z)}{1-\bar{\alpha} \varphi(z)} .
$$

Now, if $z$ belongs to an Stolz angle $\Gamma_{\beta}(\zeta)$ of vertex $\zeta$ and angle $\beta$, there exists a constant $c$ depending just on $\Gamma_{\beta}(\zeta)$ such that

$$
\left|\frac{\zeta-z}{e^{i \theta}-z}\right| \leq \frac{|\zeta-z|}{1-|z|} \leq c .
$$

Taking limit in (7) when $z \rightarrow \zeta$ for $z \in \Gamma_{\beta}(\zeta)$ and applying the Lebesgue Dominated Convergence Theorem, we deduce

$$
\int_{0}^{2 \pi} \lim _{z \rightarrow \zeta}\left(e^{i \theta}+z\right) \frac{\zeta-z}{e^{i \theta}-z} d \tau_{h, \varphi, \alpha}(\theta)=\lim _{z \rightarrow \zeta} \frac{2 \alpha h(z)(\zeta-z)}{\alpha-\varphi(z)} .
$$

Now, for $z \in \Gamma_{\beta}(\zeta)$ one has

$$
\lim _{z \rightarrow \zeta}\left(e^{i \theta}+z\right) \frac{\zeta-z}{e^{i \theta}-z}= \begin{cases}2 \zeta, & \text { if } e^{i \theta}=\zeta \\ 0 & \text { otherwise }\end{cases}
$$

so replacing in (8), one deduces

$$
\zeta \bar{\alpha} \tau_{h, \varphi, \alpha}(\{\zeta\})=\angle \lim _{z \rightarrow \zeta} h(z) \frac{\zeta-z}{\alpha-\varphi(z)} .
$$

If $\tau_{h, \varphi, \alpha}(\{\zeta\}) \neq 0$, then $\angle \lim _{z \rightarrow \zeta} \varphi(z)=\alpha$ and

$$
\angle \lim _{z \rightarrow \zeta}\left(h(z) \frac{\zeta-z}{\alpha-\varphi(z)}\right) \neq 0 .
$$

The converse follows just taking into account expression (9). 
3.1. Compactness of weighted composition operators. In this subsection, we discuss compactness of weighted composition operators in connection with the family of measures $\left\{\tau_{h, \varphi, \alpha}: \alpha \in \mathbb{T}\right\}$. Recall from Lemma 2.1 that a weighted composition operator $W_{h, \varphi}$ that is compact when acting on $\mathcal{H}^{2}$ automatically defines a compact operator on $\mathcal{H}^{1}$. Our main result in this section is in the flavor of Sarason's Theorem [16] in the setting of composition operators, who proved that $C_{\varphi}$ is compact in $\mathcal{H}^{2}$ (and therefore in $\mathcal{H}^{p}$ for any $\left.1 \leq p<\infty\right)$ if and only if $\tau_{\varphi, \alpha}^{s}=0$ for any $\alpha \in \mathbb{T}$. In the context of weighted composition operators, under the assumption that the weight $h$ is a multiplier of the space $\mathfrak{K}$ of Cauchy transforms, we have the following

Theorem 3.2. Let $W_{h, \varphi}$ be a bounded weighted composition operator on $\mathcal{H}^{2}$. Assume that $h \in \mathcal{M}(\mathfrak{K})$. Then $W_{h, \varphi}$ is compact in $\mathcal{H}^{1}$ if and only if $\tau_{h, \varphi, \alpha}^{s}=0$ for any $\alpha \in \mathbb{T}$.

The key point of the proof of Theorem 3.2 is a theorem of Goluzina, which establishes the relationship between two measures $\mu$ and $\nu$ belonging to $\mathcal{M}$, the space of all finite complex Borel measures on $\mathbb{T}$ endowed with the total variation norm. The theorem asserts that if the equation

$$
h \mathcal{K} \mu=\mathcal{K} \nu
$$

holds for some $h \in \mathcal{M}(\mathfrak{K})$, then $d \nu^{s}=h d \mu^{s}$ (see [3, Theorem 6.3.1]).

Proof. First, let us assume that $W_{h, \varphi}$ is compact in $\mathcal{H}^{1}$. Observe that

$$
\mathcal{K} \tau_{h, \varphi, \alpha}=h \mathcal{K} \tau_{1, \varphi, \alpha}
$$

for any $\alpha \in \mathbb{T}$. Since $h \in \mathcal{M}(\mathfrak{K})$, Goluzina's Theorem (see [3, Theorem 6.3.1]) will ensure that $\tau_{h, \varphi, \alpha}^{s}=0$ for any $\alpha \in \mathbb{T}$ as far as we prove that $\tau_{1, \varphi, \alpha}^{s}=0$ for any $\alpha \in \mathbb{T}$. This will be accomplished by proving that $W_{1, \varphi}=C_{\varphi}$ is compact on $\mathcal{H}^{1}$ (and therefore, by Sarason's Theorem, the result will follow). Note that, automatically, $C_{\varphi}$ will be compact on $\mathcal{H}^{p}$ for any $1 \leq p<\infty$.

We proceed by contradiction. Assume $C_{\varphi}$ is not compact on $\mathcal{H}^{1}$. Then, there exists $\left\{w_{n}\right\} \subset \mathbb{D}$ such that $\left|w_{n}\right| \rightarrow 1$, but

$$
\lim _{n \rightarrow \infty}\left\|C_{\varphi} \frac{1-\left|w_{n}\right|^{2}}{\left(1-\overline{w_{n}} z\right)^{2}}\right\|_{1} \neq 0
$$

(see [15], for instance). So, there exists $\varepsilon>0$ and a subsequence $\left\{w_{n_{k}}\right\} \subset \mathbb{D}$ such that

$$
\lim _{k \rightarrow \infty}\left\|C_{\varphi} \frac{1-\left|w_{n_{k}}\right|^{2}}{\left(1-\overline{w_{n_{k}}} z\right)^{2}}\right\|_{1} \geq \varepsilon
$$

On the other hand, since $W_{h, \varphi}$ is compact in $\mathcal{H}^{1}$ by hypotheses, it follows that

$$
\lim _{k \rightarrow \infty}\left\|W_{h, \varphi} \frac{1-\left|w_{n_{k}}\right|^{2}}{\left(1-\overline{w_{n_{k}}} z\right)^{2}}\right\|_{1}=0
$$

(see [5], for instance). Hence, for any $\delta>0$, one deduces that

$$
\lim _{k \rightarrow \infty}\left(1-\left|w_{n_{k}}\right|^{2}\right) \int_{\{\xi \in \mathbb{T}:|h(\xi)|>\delta\}} \frac{|h(\xi)|}{\left|1-\overline{w_{n_{k}}} \varphi(\xi)\right|^{2}} d m(\xi)=0,
$$


and this is a contradiction, because $h \in \mathcal{H}^{\infty}$ and thus it is non-zero almost everywhere. Therefore, $C_{\varphi}$ is compact on $\mathcal{H}^{1}$ and the implication follows.

In order to prove the converse, let us assume $\tau_{h, \varphi, \alpha}^{s}=0$ for any $\alpha \in \mathbb{T}$. Once again, from the observation

$$
\mathcal{K} \tau_{h, \varphi, \alpha}=h \mathcal{K}_{\tau_{1, \varphi, \alpha}}
$$

for any $\alpha \in \mathbb{T}$ along with the facts that $h \in \mathcal{M}(\mathfrak{K})$ and Goluzina's Theorem (see $[3$, Theorem 6.3.1]), we may ensure that $\tau_{1, \varphi, \alpha}^{s}=0$ for any $\alpha \in \mathbb{T}$. In other words, $W_{1, \varphi}=C_{\varphi}$ is compact on $\mathcal{H}^{p}$ for any $1 \leq p<\infty$.

In order to prove that $W_{h, \varphi}$ is compact in $\mathcal{H}^{1}$, it is enough to show that

$$
\left\|W_{h, \varphi} \frac{1-|a|^{2}}{(1-\bar{a} z)^{2}}\right\|_{1} \rightarrow 0
$$

as $|a| \rightarrow 1$ (see [5], for instance). By the Cauchy-Schwarz inequality, one has

$$
\left\|W_{h, \varphi} \frac{1-|a|^{2}}{(1-\bar{a} z)^{2}}\right\|_{1} \leq\left\|\frac{\left(1-|a|^{2}\right)^{1 / 2} h(z)}{1-\bar{a} \varphi(z)}\right\|_{2}\left\|\frac{\left(1-|a|^{2}\right)^{1 / 2}}{1-\bar{a} \varphi(z)}\right\|_{2}
$$

which tends to zero as $|a| \rightarrow 1$ since $W_{h, \varphi}$ is bounded in $\mathcal{H}^{2}$ and $C_{\varphi}$ is compact on $\mathcal{H}^{2}$. This proves the converse, and therefore Theorem 3.2.

Remark 3.3. If the weight $h$ is not a multiplier of the space $\mathfrak{K}$ in Theorem 3.2, then are self-maps $\varphi$ of the disc for which we cannot introduce the measures $\tau_{h, \varphi, \alpha}$ as in Definition 2.1 For instance, take

$$
h(z)=\exp \left(\frac{z+1}{z-1}\right) .
$$

It holds that $h \notin \mathcal{M}(\mathfrak{K})$ (see [3, Theorem 6.6.11]). Consider $\varphi(z)=z$. Then $W_{h, \varphi}=T_{h}$, which is clearly bounded on all $\mathcal{H}^{p}$ spaces, whereas $W_{h, \varphi}^{*}=T_{\bar{h}}$, which is not bounded on $\mathcal{A}(\mathbb{D})$.

\section{ACKNOWLEDGEMENT}

The authors are grateful to the referee for many useful comments.

\section{REFERENCES}

[1] A. B. Aleksandrov, Multiplicity of boundary values of inner functions, Izv. Akad. Nauk Armyan. SSR Ser. Mat. 22 (1987), no. 5, 490-503.

[2] J. A. Cima and A. L. Matheson, Cauchy transforms and composition operators, Illinois J. Math. 42 (1998), no. 1, 58-69.

[3] J. A. Cima, A. L. Matheson and W. T. Ross, The Cauchy transform, Mathematical Surveys and Monographs, 125. American Mathematical Society, Providence, RI, 2006.

[4] C. Cowen, Linear fractional composition operators on $H^{2}$, Integral Equations Operator Theory 11 (1988), no. 2, 151-160. 
[5] Z. C̆učković and R. Zhao, Weighted composition operators between different weighted Bergman spaces and different Hardy spaces, Illinois J. Math. 51 (2007), no. 2, 479-498.

[6] S. P. Eveson, Compactness criteria for integral operators in $L^{\infty}$ and $L^{1}$ spaces, Proc. Amer. Math. Soc. 123 , no. 12 (1995), 3709-3716.

[7] E. A. Gallardo-Gutiérrez, M. J. González, P. J. Nieminen and E. Saksman, On the connected component of compact composition operators on the Hardy space, Advances in Math. 219 (2008), no. 3, 986-1001.

[8] J. B. Garnett, Bounded analytic functions, Revised first edition. Springer-Verlag, New York, 2007.

[9] J. E. Littlewood, On inequalities in the theory of functions, Proc. London Math. Soc. (2) 23 (1925), 481-519.

[10] A. Matheson and M. Stessin, Applications of spectral measures, Recent Advances in OperatorRelated Function Theory, Contemp. Math. 393 (2006), 15-27.

[11] R. Nevanlinna, Remarques sur le lemme de Schwarz, Comptes Rendus Acad. Sci. Paris 188 (1929), $1027-1029$.

[12] P. J. Nieminen and E. Saksman, Boundary correspondence of Nevanlinna counting functions for self-maps of the unit disc, Trans. Amer. Math. Soc. 356 (2004), no. 8, 3167-3187

[13] P. J. Nieminen and E. Saksman, On compactness of the difference of composition operators, J. Math. Anal. Appl. 298 (2004), 501-522.

[14] A. Poltoratski and D. Sarason, Aleksandrov-Clark measures, Recent Advances in Operator-Related Function Theory, Contemp. Math. 393 (2006), 1-14.

[15] E. Saksman, An elementary introduction to Clark measures, Topics in Complex Analysis and Operator Theory, Univ. Málaga, 2007, pp. 85-136.

[16] D. Sarason, Composition operators as integral operators, Analysis and Partial Differential Equations, Lecture Notes in Pure and Appl. Math., vol. 122, Dekker, New York, 1990, pp. 545-565.

[17] J. H. Shapiro and C. Sundberg, Compact composition operators on $L^{1}$, Proc. Amer. Math. Soc. 108, no. 2 (1990), 443-449.

Departamento de Análisis Matemático, Facultad de Ciencias Matemáticas, Universidad Complutense de Madrid e iUma, Plaza de Ciencias 3, 28040 Madrid, SPain.

E-mail address: eva.gallardo@mat.ucm.es

School of Mathematics, University of Leeds, Leeds LS2 9JT, U.K.

E-mail address: J.R.Partington@leeds.ac.uk 